\title{
CONTRIBUTION \\ A L'ÉTUDE DE LA DIFFÉRENCIATION DES SPERMATOZOÏDES MORTS ET DES SPERMATOZOÏDES VIVANTS DANS LE SPERME DE TAUREAU
}

PAR

\author{
R. ORTAvant, Suzanne DUPONT, H. PAUThe, G. ROUSSEL \\ Station de Physiologie animale, Centre National de Recherches Zootechniques, \\ Jouy-en-Josas $\left(^{(1)}\right.$
}

Le développement de l'Insémination artificielle chez les animaux domestiques a montré la nécessité d'un diagnostic rapide et sûr de la valeur du sperme.' De très nombreux tests ont été proposés ; la détermination de la proportion relative des spermatozoïdes morts est l'un des plus utiles.

LASLEY, EASLEY et MAC KENZIE (I942) proposèrent une technique de coloration modifiée par LASLEY et BOGART (I943), grâce à laquelle, seuls les spermatozoïdes morts étaient colorés par un mélange de bleu opale et d'éosine. Puis Shaffer et Almouist (I948) remplacèrent le bleu opale, colorant qu'il est difficile de se procurer, par le bleu d'aniline. On pouvait ainsi facilement déterminer les pourcentages de spermatozoïdes morts dans un échantillon de sperme.

Cependant on est en droit de se demander, si, au cours des opérations de confection des frottis (coloration, étalement, fixation), des spermatozoïdes vivants ne sont pas tués. La connaissance de ces causes éventuelles d'erreur peut permettre de déterminer les conditions d'application pratique de cette méthode et la sécurité qu'elle offre. Malheureusement les travaux cités ne fournissent pas suffisamment de précision dans ce sens ; c'est pourquoi nous en avons repris l'étude.

Le colorant utilisé fut celui indiqué par SHAFFER et AlMQUIS'T (I948) :

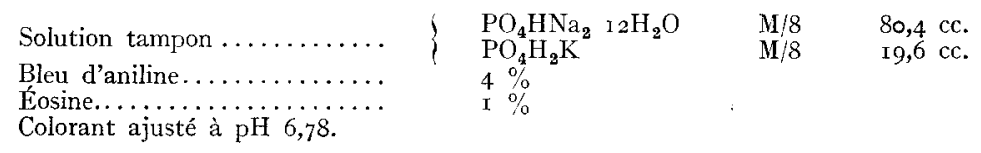

(1) Ce travail a été effectué en partie au Laboratoire de Zootechnie de l'École Nationale d'Agriculture de Rennes. 
Le sperme fut récolté sur 3 taureaux Normand et Pie-Noir breton à l'aide d'un vagin artificiel. Les frottis furent effectués selon la méthode par entraînement indiquée par SalisbURy, WiLETT et SELIGMANN (I942), légèrement modifiée.

\section{RESULTATS}

\section{$I^{0}$ Influence de l'observateur}

Dans cette technique la tête des spermatozoïdes morts est colorée en pourpre avec une intensité variable, la partie antérieure étant parfois plus pâle et même non colorée. Les spermatozoïdes vivants ne sont pas pénétrés par le colorant et apparaissent en clair sur un fond bleu sombre. Cependant il arrive parfois, lorsque les frottis sont épais, que le fond bleu assombrisse la tête des spermatozoïdes vivants et puisse faire croire à des spermatozoïdes morts. En réalité, ils n'ont pas été pénétrés par le colorant.

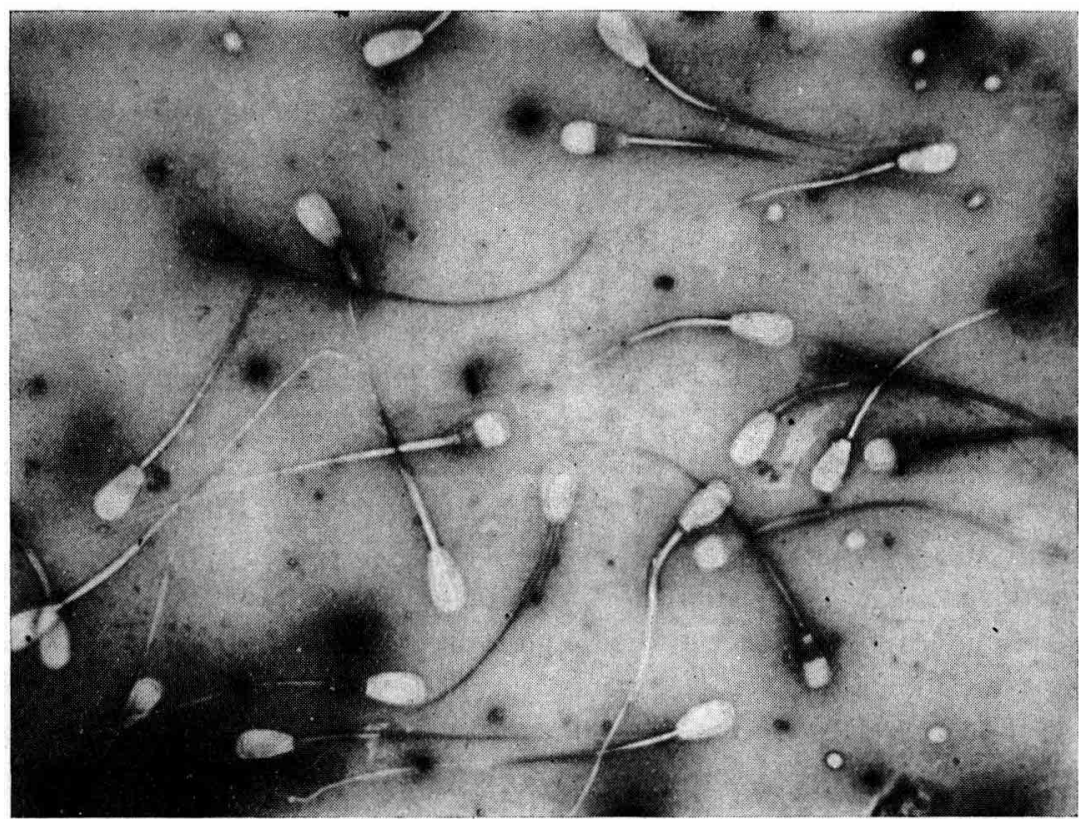

Microphotographie : frottis de spermatozoïdes de Taureau colorés au bleu d'aniline-éosine. Les spermatozoïdes vivants sonts absolument incolores.

Il était donc important de vérifier si la différenciation des spermatozoïdes vivants et morts était indépendante de l'observateur. A cet effet, deux obser. vateurs différents effectuèrent les déterminations des pourcentages des spermatozoïdes colorés sur les mêmes frottis (29) selon la même technique de prospection. 
Les résultats sont résumés dans le tableau I.

\section{TABleau I}

Influence de l'observateur

\begin{tabular}{c|c|c}
\hline Observateur & $\%$ Spz colorés & Écart-type \\
\hline A & 29,7 \\
B & 28,9 & $\pm 7,4$ \\
\hline
\end{tabular}

Erreur type de la moyenne des écarts : + o,66

Ecart-type des écarts : $\pm 2,5$

$t=\mathrm{I}, 22$ d'où $0,2<\mathrm{P}<0,3$

La différence de $0,8 \%$ entre les résultats des deux observateurs n'est pas significative : elle est très probablement le fait du hasard. La valeur numérique trouvée par cette méthode est donc indépendante de 1'observateur. Ceci confirme les résultats de LASLEY, EASLEY et MC KENZIE (I942) utilisant le bleu opale.

\section{$2^{\circ}$ Étude de l'homogénéité des frottis}

On pouvait se demander si la répartition des spermatozoïdes colorés dans les frottis était homogène.

Des comptages systématiques furent donc effectués à la base, au milieu et au sommet des frottis dans le sens longitudinal, sur les deux bords et au milieu dans le sens transversal.

Les résultats figurent dans les tableaux II et III.

TABLEAU II

Influence de la localisation longitudinale.

\begin{tabular}{|c|c|c|c|}
\hline Spermatozoïdes Localisation & Base & Milieu & Sommet \\
\hline Colorés & I 3.423 & I 4.426 & I 5.265 \\
\hline Total & 40.472 & 40.772 & $4 \mathrm{I} \cdot \mathrm{I} 55$ \\
\hline$\%$. & $33,16 \%$ & $35,37 \%$ & $37,09 \%$ \\
\hline
\end{tabular}

La différence entre les pourcentages calculés au milieu et au sommet est hautement significative, de même que celle entre les pourcentages calculés à la base et au milieu.

Il y a donc une augmentation progressive du pourcentage des spermatozoïdes colorés en allant vers le sommet du frottis. Ce fait provient-il d'un entrâ̂- 
nement des spermatozoïdes morts par le bord de la lamelle ou d'un effet nocif dû à la pression exercée par celle-ci lors de l'étalement ? La première hypothèse est confirmée par l'expérience suivante : un nombre connu de spermatozoïdes tués par chauffage est mélangé en proportions variées avec certaines quantités de spermatozoïdes vivants. Le pourcentage de spermatozoïdes colorés se trouve inférieur au pourcentage réel de spermatozoïdes morts au départ des frottis, alors qu'à l'arrivée, il lui est supérieur, les agglutinats de spermatozoïdes morts étant concentrés principalement à cet endroit du frottis. Il y a donc bien entraînement des spermatozoïdes morts par l'étalement.

TABLEAU III

Influence de la localisation transversale.

\begin{tabular}{|c|c|c|c|}
\hline Spermatozoïdes & Bord I $\left(b_{1}\right)$ & Milieu (m) & Bord $2\left(b_{2}\right)$ \\
\hline Colorés. & I 4.404 & I $3.73 \mathrm{I}$ & 14.978 \\
\hline Total ..... & 41.262 & 40.206 & 40.931 \\
\hline$\% \ldots \ldots \ldots \ldots$ & 34,9 & 34, I 5 & $3^{6,59}$ \\
\hline
\end{tabular}

Dans le sens transversal, c'est au milieu du frottis que le pourcentage de spermatozoïdes colorés est le plus faible. Les différences sont moins nettes que précédemment, mais cependant significatives (entre $b_{1}$ et $m, x^{2}=5, \mathrm{P}=0,03$ ).

Ce fait s'explique sans doute pour la même raison que précédemment, la goutte de sperme étant déposée au milieu.

Les frottis devront donc être prospectés selon une diagonale pour que le résultat soit représentatif de l'échantillon examiné.

\section{$3^{\circ}$ Nombre de spermatozoïdes a compter par frottis}

Nous avons d'abord effectué nos numérations sur 2 ooo spermatozoïdes ce qui représentait un travail de 40 minutes par frottis et, par conséquent, exigeait un temps trop long pour la pratique de l'insémination artificielle.

Il était donc nécessaire de chercher la limite inférieure du nombre des spermatozoïdes à compter pour obtenir un pourcentage statistiquement représentatif.

Sur 36 frottis, les résultats furent respectivement calculés en comptant 2 ooo spermatozoïdes répartis dans tout le frottis, 600 et 550 spermatozoïdes prélevés sur une diagonale du frottis. 
TABLEAU IV

Influence du nombre de spermatozoïdes comptés

\begin{tabular}{c|c|c}
\hline Caractéristiques Statistiques & $\begin{array}{c}\text { Entre } \% \text { sur } 2000 \mathrm{spz} \\
\text { et } \% \text { sur } 600 \mathrm{spz}\end{array}$ & $\begin{array}{c}\text { Entre } \% \text { sur } 2000 \mathrm{spz} \\
\text { et } \% \text { sur } 150 \mathrm{spz}\end{array}$ \\
\hline Coefficient de corrélation......... & $r_{\mathbf{1}}=+0,99$ & $r_{\mathbf{2}}=+0,97$ \\
\hline Déviation standard des écarts .... & $\sigma_{\mathbf{1}}= \pm \mathbf{1 , 4 7}$ & $\sigma_{\mathbf{2}}= \pm 2,23$ \\
\hline \hline
\end{tabular}

Les coefficients de corrélation entre les pourcentages calculés sur 2000 spermatozoïdes et ceux calculés sur 600 et I 50 spermatozoïdes sont très voisins de l'unité, les déviations standards des écarts étant inférieures à $2,5 \%$.

Il semble donc que, dans la pratique courante, il suffise de compter I5o spermatozoïdes prélevés sur une diagonale du frottis, ce qui représente un gain de temps considérable puisque le contrôle d'un frottis exige alors moins de 5 minutes.

Ces résultats confirment ceux de SALISBURy et MERciER (I945) concernant le calcul des pourcentages de spermatozoïdes anormaux.

\section{$4^{\circ}$ Influence de la température de confection des frottis et de la durée d'action du colorant}

La température à laquelle les frottis sont effectués, a une influence sur le pourcentage des spermatozoïdes colorés. LASLEY, EASLEY et MC KENZIE (1942) avaient montré qu'à $37^{\circ}$ et à $o^{\circ}$ on obtenait le plus de spermatozoïdes colorés. A partir des mêmes échantillons de sperme, des frottis furent effectués aussitôt après la récolte à $15^{\circ} \mathrm{C}$ et $28^{\circ} \mathrm{C}$, le colorant agissant pendant des temps variés.

\section{TABLEAU $\mathrm{V}$}

Influence de la température en fonction de la durée d'action du colorant sur le pourcentage de spermatozoïdes colorés

\begin{tabular}{|c|c|c|c|c|}
\hline Durée d'action & I $5^{\circ}$ & $28^{\circ}$ & \lrcorner & Nombre de frottis \\
\hline $\begin{array}{l}\text { 30 secondes } \ldots \ldots \\
\text { I minute...... } \\
2 \text { minutes } \ldots \ldots \ldots \\
5 \text { minutes } \ldots \ldots\end{array}$ & $\begin{array}{l}26,2 \% \\
41,0 \% \\
46,3 \% \\
52,8 \%\end{array}$ & $\begin{array}{l}\text { 1 } 7,6 \% \\
\text { 18,8 } \% \\
21,8 \% \\
28,0 \%\end{array}$ & $\begin{array}{cc}8,6 & \text { significatif } \\
22,6 & " \\
24,5 & " \\
24,8 & "\end{array}$ & $\begin{array}{l}22 \\
22 \\
22 \\
22\end{array}$ \\
\hline
\end{tabular}

Ainsi la température a une action significative sur le pourcentage de spermatozoïdes colorés, même pour des durées d'action du colorant de 30 secondes. Lorsque les frottis sont faits à la température de $I 5^{\circ}$, le pourcentage des sper- 
matozoïdes colorés est plus élevé que lorsqu'ils sont effectués à $28^{\circ}$, l'action de la température sur la mortalité des spermatozoïdes étant d'autant plus marquée que la durée d'action est plus longue.

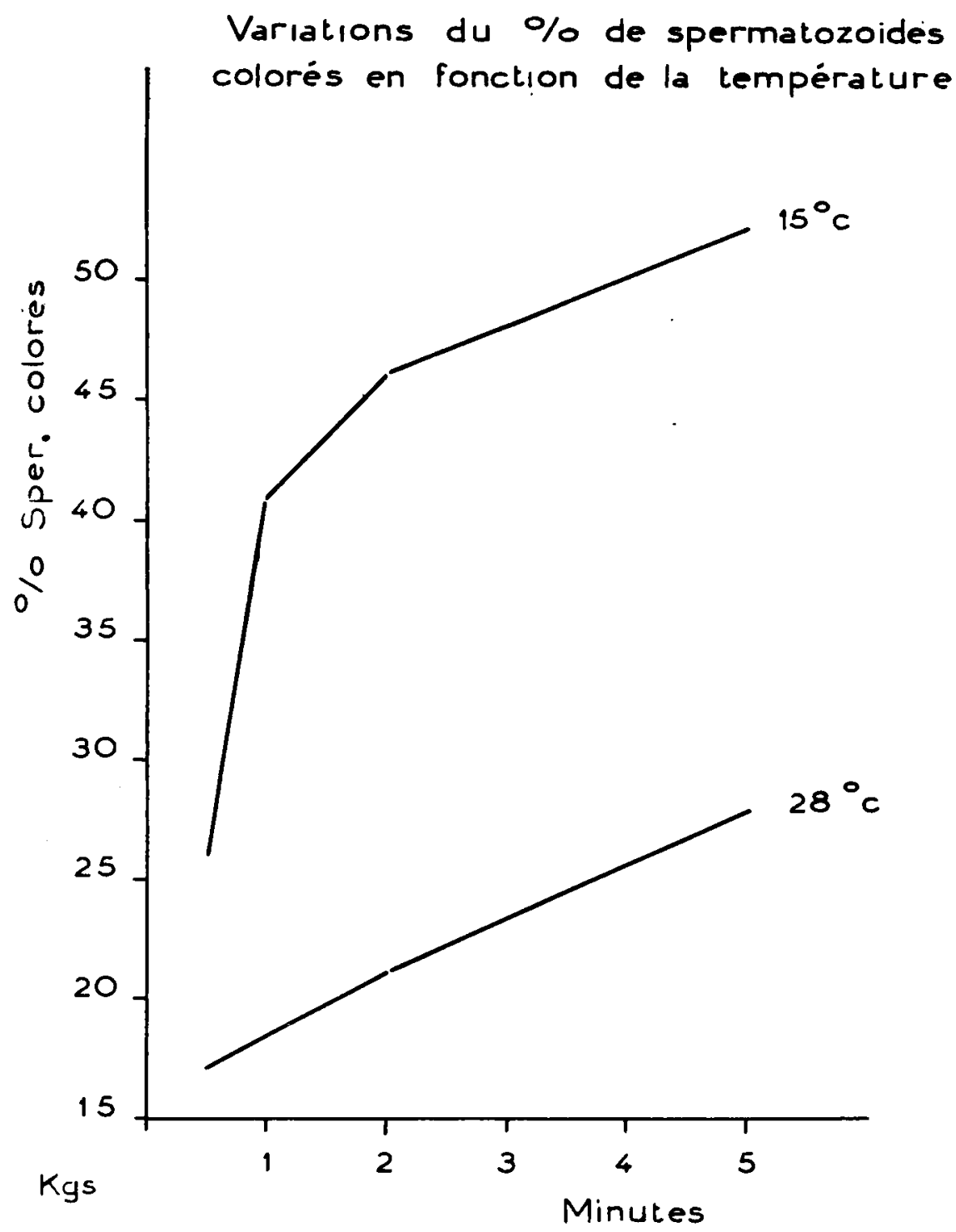

Ceci nous montre que l'action des températures basses $\left(15^{\circ}\right)$ sur du sperme en couche mince est extrêmement rapide, puisqu'après une minute le pourcentage des spermatozoïdes morts est presque aussi élevé que celui obtenu après une durée d'action de 5 minutes. Il existe d'ailleurs de grandes différences individuelles de sensibilité parmi les divers spermes. 
Pendant combien de temps doit-on alors laisser agir le colorant à $28^{\circ}$ pour obtenir un frottis facilement lisible avec le minimum d'erreur ?

Les résultats inscrits dans le tableau $\mathrm{V}$ nous montrent qu'à $28^{\circ}$ le pourcentage des spermatozoïdes colorés augmente au fur et à mesure que l'action du colorant se prolonge. Mais cette augmentation se produit selon une loi sensiblement linéaire, contrairement à ce qui se passe à $I 5^{\circ}$. Cette action est vraisemblablement due à une action propre légèrement toxique du colorant.

Il était alors intéressant de savoir si des temps inférieurs à 30 secondes permettaient d'obtenir une diminution importante des pourcentages de spermatozoïdes colorés. Des essais furent effectués pour des temps de ro secondes. Mais à $28^{\circ}$ la différence était inférieure à I \% et par conséquent pratiquement négligeable. Etant donné la difficulté à réaliser des frottis convenables avec une durée d'action de ro secondes, le temps de 30 secondes doit être adopté.

\section{$5^{\circ}$ Influence de la température de séchage des frottis}

LASLEY, EASIEY et MAC KENZIE (I942) laissaient sécher leurs frottis à l'air, par contre SHAFFER et ALMQUIST (I946) préconisent de sécher les frottis à haute température avec un ventilateur. La réalisation du séchage des frottis aux températures léthales est difficile car il doit être instantané, sinon il se produit une augmentation du nombre des spermatozoïdes morts. Ainsi lorsque le frottis est trop épais, tous les spermatozoïdes se colorent, le frottis mettant trop longtemps à sécher. Il était donc nécessaire de reprendre l'étude de cette question.

\section{TABLEAU VI}

Influence de la température de séchage

\begin{tabular}{|c|c|c|c|c|}
\hline 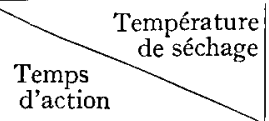 & $28^{\circ}-30^{\circ}$ & $I 40^{\circ}-170^{\circ}$ & $\begin{array}{l}\text { Nombre } \\
\text { de frottis }\end{array}$ & $\begin{array}{l}\text { Interprétation } \\
\text { statistique }\end{array}$ \\
\hline $\begin{array}{l}30 \text { secondes......... } \\
5 \text { minutes.......... }\end{array}$ & $\begin{array}{l}16,4 \% \\
26,2 \%\end{array}$ & $\begin{array}{l}17,7 \% \\
27,9 \%\end{array}$ & $\begin{array}{l}25 \\
25\end{array}$ & $\left\{\begin{array}{c}t=\mathrm{I}, \mathrm{Io} ; 0,2<\mathrm{P}<0,3 \\
\text { non significatif } \\
t=0,56 ; 0,5<\mathrm{P}<0,6 \\
\text { non significatif }\end{array}\right.$ \\
\hline & $28^{\circ}-30^{\circ}$ & $4^{60}-70^{0}$ & & \\
\hline $\begin{array}{l}30 \text { secondes........ } \\
5 \text { minutes.......... }\end{array}$ & $\begin{array}{l}19,5 \% \\
29,3 \%\end{array}$ & $\begin{array}{l}\mathbf{1} 8,5 \% \\
28,9 \%\end{array}$ & $\begin{array}{l}26 \\
23\end{array}$ & $\left\{\begin{array}{l}t=1,02 ; 0,3<\mathrm{P}<0,4 \\
\text { non significatif } \\
t=0,34 ; 0,7<\mathrm{P}<0,8 \\
\text { non significatif }\end{array}\right.$ \\
\hline
\end{tabular}

Le séchage des frottis à $1^{\prime}$ air libre à $28^{\circ}-30^{\circ}$ donne moins de spermatozoïdes colorés que le séchage à très haute température, mais un peu plus que celui à température moyenne. Dans aucun cas la différence n'est significative. Cepen- 
dant comme la différence est faible ( $\leqslant \mathrm{I} \%$ dans le dernier cas) on peut effectuer le séchage des frottis aux températures de $28^{\circ}-30^{\circ}$ sans risque d'erreur importante.

\section{RÉSUME ET CONCLUSIONS}

Des essais ont été effectués pour déterminer l'influence de différents facteurs sur la mise en évidence des spermatozoïdes morts, dans du sperme de taureau, par coloration élective. Le colorant utilisé a été un mélange de bleu d'aniline $(4 \%)$ et d'éosine ( $(\%)$ dans une solution de phosphate tamponnée à $\mathrm{pH}=6,78$.

Io Il n'existe pas de différence significative entre les résultats obtenus par deux observateurs effectuant la détermination des pourcentages de spermatozoïdes colorés sur les mêmes frottis. Cette technique peut donc être utilisée par toute personne déjà entraînée à l'observation des spermatozoïđes.

$2^{\circ}$ Il y a une augmentation progressive du nombre des spermatozoïdes colorés en allant de la base au sommet du frottis dans le sens longitudinal et du milieu vers les bords, dans le sens transversal. L'observateur devra donc effectuer son échantillonnage selon une diagonale du frottis pour obtenir un pourcentage représentatif.

$3^{\circ}$ Il suffit de compter un total de I5o spermatozoildes par frottis pour obtenir un résultat statistiquement représentatif de celui-ci. Ce test est donc rapide.

$4^{\circ}$ La température ambiante et la température d'action du colorant ont une influence très importante sur les résultats. C'est à $28^{\circ}$, le colorant agissant pendant 30 secondes, que l'action de ces facteurs est minimum. A I $5^{\circ}$ on obtient tune forte augmentation des pourcentages de spermatozoïdes colorés, d'autant plus que la durée d'action du colorant est plus longue.

$5^{\circ}$ Les frottis peuvent être séchés à $28^{\circ}-30^{\circ} \mathrm{C}$ aussi bien qu'à haute température ( $140-\mathrm{I}_{7} 0^{\circ} \mathrm{C}$.)

Ainsi cette technique facile peut être utilisée par les Centres d'insémination artificielle, en tenant compte des conditions discutées ci-dessus.

\section{BIBLIOGRAPHIE}

Lasley (F. F.), EAsley (G. T.), MC Kongrie (E.F.). - A staining method for the differenciation of live and dead spermatozoa. I. Applicability to the staining for ram spermatozoa. Anat. Rec.,1942, 82, 2, pp. 167-174.

LASLey (F. F.), Bogard (R.). - Some factors influencing reproductive efficiency of range cattle under artificial and natural breeding conditions. Mo. Agric. Exp. Sta. Res. Bull., I $943,376, \mathrm{pp} . \mathrm{I} 3$.

SHAFFER (H. E.), ALMQUisT (J. O.). - - Vital staining of bovine spermatozoa with an eosinanilin blue staining mixture. J. Dairy Sci., I948, 31, 8, pp. 668-677.

Salisbury (G. W.), Willett (E. L.), Seligman (J.). - The effect of the method of making semen smears upon the number of morphologically abnormal spermatozoa. J. of Anima Sci., I942, I, I, pp. 200.5. 